\title{
Development of the Neonatal Rat Small Intestinal Barrier to Nonspecific Macromolecular Absorption: Effect of Early Weaning to Artificial Diets ${ }^{1}$
}

\author{
SAUL TEICHBERG, ERIKA ISOLAURI, RAUL A, WAPNIR, \\ BETH ROBERTS. AND FIMA LIFSHITZ \\ Departments of Pediatrics and Laboratories, North Shore University Hospilal-Cornell University Medical \\ College. Manhasset, New York 11030
}

\begin{abstract}
We studied the effect of early weaning from maternal breast milk to artificial diets on rat jejunal absorption of an exogenous $40-\mathrm{kD}$ glycoprotein, horseradish peroxidase (HRP). Rat pups, fed maternal milk (MM) from birth, received one of three diets for the last 4 d before weaning (d 17-21): MM, protein hydrolysate formula (PH), or soy formula (S). Some rats were pretreated on d 14 with intraperitoneal hydrocortisone (5 mg/rat). In MM-fed rat pups, jejunal HRP absorption was markedly higher on $d 17$ than on d 21. [Geometric means (95\% confidence interval) were: $d$ 17, $626.4(461.3,850.6)$ versus d $21,90.6(48.2,170.5) \mathrm{IU} \mathrm{HRP} / \mathrm{mL} \times \mathrm{cm} \times \mathrm{min}, p<$ 0.01.] By contrast, 21-d-old PH- and S-fed pups maintained elevated absorption of the tracer $[\mathrm{PH}, 292.3(177.5,480.6)$, $p<0.05$ versus $\mathrm{MM}$ pups, S, 340.8 (164.4, 704.8), $p<$ 0.01 versus MM pups]. Wt-matched control studies indicate that the difference in HRP absorption was not due to the smaller body wt of formula-fed pups. The increased absorption in formula-fed animals was suppressed by hydrocortisone. In S-fed pups, the increased macromolecular absorption appeared, in part, to be the result of diffusion across altered villus absorptive cells. In PH-fed pups, there was no evidence of damage and HRP absorption appeared to occur by vesicle-mediated transport. Delay in the normal maturation of small intestinal "closure" appears to be associated with early weaning to artificial diets. This may lead to increased nonspecific macromolecular permeability that could result in immune-mediated sensitization and food intolerance. (Pediatr Res 28: 31-37, 1990)
\end{abstract}

Abbreviations

MM, matemal milk

PH, profein hydrolysate formula

S, soy formula

HRP, horseradish peroxidase

$\mathrm{HC}$, hydrocortisone

The "closure" of the neonatal mammalian small intestinal epithelium to the lumen-to-blood translocation of macromolecules is an incompletely understood major physiologic process

Received September 18, 1989, accepted March 19, 1990.

Correspondence: Saul Teichberg, Ph.D., Electron Microscopy Laboratory, North Shore University Hospital, Manhasset. NY 11030

Supported in part by PHS Grant RR05924-05.

'Presented in part at the 1989 Annual Meeting of the American Pediatric Society/Society for Pediatric Research, Washington, DC, May 1989.
(1-4). The timing and pattern of closure varies between species, as a function of neonatal maturity and intestinal development at birth $(4,5)$. In altricial species, such as the rat, the gut remains open to the uptake of nutritional, immunologic, and growthpromoting trophic macromolecules from MM for a relatively long time (21 d) after birth. In precocial species, such as the guinea pig, closure occurs earlier (2-4 d) (4). Macromolecular absorption in altricial species, such as the rat, occurs by receptormediated and soluble endocytosis. This is reflected in the morphology of the absorptive epithelial cells that are filled with endocytotic tubules, vesicles, and larger vacuoles that largely disappear at closure (4). Human infants at birth are considered intermediate between altricial and precocial species at birth; MM-derived immunologic factors are beneficial and MM hormonal-trophic factors may have an as yet undefined physiologic role $(6,7)$.

Much remains to be learned about the immunologic, trophic, hormonal, and nutritional factors that may regulate closure and the mechanisms that delay this process $(8-12)$. The effects of diet and nutritional factors on the normal development of the gastrointestinal macromolecular barrier are incompletely understood. Formula feedings, in contrast to MM, have been reported to lead to increases in intestinal permeability in human infants, newborn rabbits, and guinea pigs $(11,12)$. In humans, the use of small molecule, nonmetabolizable markers limits the relevance to macromolecular absorption (12-14). In the rabbit, no artificial-feeding permeability-damaging factor has been isolated, and no immunologic mechanism accounting for the increased absorption demonstrated (11). Although it could turn out that normal closure requires the continuous presence of MM-derived trophic or hormonal factors, no experimental evidence for such a mechanism has been reported (7). Some evidence does suggest a possible role for endogenous glucocorticoids in this process in rats $(1,9,10,15,16)$.

We evaluated the effect of early weaning to formula feedings (PH or S), as well as the effects of $\mathrm{HC}$ administration, on neonatal rat small intestinal closure to a nonspecific, $40-\mathrm{kD}$ macromolecule, HRP. The studies were done during the last $4 \mathrm{~d}$ before weaning ( $d$ 17-21), when the pups feed independently on formulas and do not require the dam for thermoregulation or bowel function. Feeding either PH or S prevented normal jejunal closure to absorption of HRP, whereas pretreatment of pups with $\mathrm{HC}$ led to HRP absorption like that seen in MM pups. Our observations lend support to the view that normal closure is dependent on the continuous presence of $\mathrm{MM}$ factors and that premature weaning to artificial diets may lead to increased nonspecific macromolecular permeability. 


\section{MATERIALS AND METHODS}

Animals and Experimental Feedings. Newborn male and female rat pups, Wistar strain [Crl:(WI)BR, Charles River Labs, Wilmington, MA] were reared at a density of $12 \mathrm{pups} / \mathrm{dam}$ until $17 \mathrm{~d}$ of age, under natural, MM feeding conditions. On $\mathrm{d} 17$, the pups were divided into three feeding groups of four rats each for $4 \mathrm{~d}$, until d 2 l of age. One group remained with the mother and was breast fed with $\mathrm{MM}$, one group was fed ad libitum with a $\mathrm{S}$ liquid commercial diet (Isomil-R), and the third group was fed ad libitum with a PH formula (Nutramigen-R). The formulas were delivered by standard water bottles to which the rat pups adapted readily. An effort was made to distribute the rat pups equally by sex and wt among the three feeding groups. The pups were all kept in metabolic cages with wire mesh bottoms to avoid coprophagy. A folded paper diaper was placed in half of the cage to help pups with control of body temperature. The pups were weighed daily and the effect of feedings on wt gain monitored. Figure 1 outlines the basic experimental feeding protocol. All animal experimentation was approved by the institutional Animal Care Committee and was performed in accordance with the guidelines set forth in the NIH Guide for the Care and Use of Laboratory Animals (17).

Because our initial data indicated that MM-fed rat pups had a small but significant increase in wt after $4 \mathrm{~d}$ as compared with formula fed pups (Table 1), a wt-matched control study was carried out. For this purpose, litter density of MM-fed animals was maintained at $12 \mathrm{pups} / \mathrm{dam}$ before and during the 17- to $21-d$ experimental feeding period. MM-fed rat pups, selected from this litter and wt-matched to formula-fed pups, were then studied at $\mathbf{d} 21$.

The normal development of closure to HRP between 17 and 21 was also evaluated in several litters of rat pups. This was done

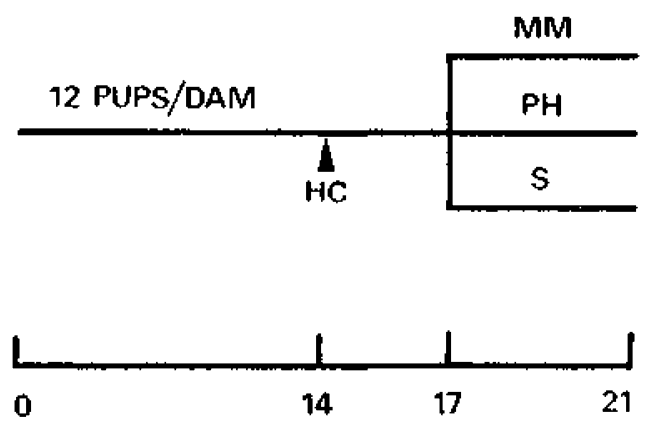

\section{AGE (DAYS)}

Fig. 1. Diagram of the basic experimental feeding sequence. Five mg $\mathrm{HC} /$ pup were administered intraperitoneally on d 14. During the $4 \mathrm{~d}$ experimental feeding period, from d 17-21 of age, there were 4 pups/ treatment.

Table 1. Effect of diet and hydrocortisone treatment on rat pup wt

\begin{tabular}{lcccc}
\hline \multicolumn{1}{c}{ Diet } & $n$ & g/pup & $p v_{s}$ MM & $p v_{s}$ HC-free \\
\hline MM & 24 & $52.6+0.9$ & & \\
PH & 24 & $48.8+0.8$ & 0.05 & \\
S & 24 & $45.7+0.7$ & 0.05 & \\
PH + HC & 13 & $41.3+1.1$ & 0.01 & 0.05 \\
S + HC & 12 & $38.5+0.9$ & 0.01 & 0.05 \\
\hline
\end{tabular}

Rat pup wt on d 21, after the experimental feeding period with either $\mathrm{MM}, \mathrm{PH}$, or $\mathrm{S}$ and with or without HC pretreatment. Initial wt of the rat pups on $\mathrm{d} 17$ in the three experimental feeding groups was indistinguishable (MM, $43.2 \pm 0.67 ; \mathrm{PH}, 42.8 \pm 0.69 ; \mathrm{S}, 41.5 \pm 0.50 \mathrm{~g} / \mathrm{pup}$ ). All pups gained $w$ t over the $4 \cdot d$ experimental period. Values are arithmetic means + SEM. Statistical analysis was by one-way analysis of variance. with paired contrasts by Tukey's test. $n=$ no. pups/group. by comparing jejunal HRP absorption at $d 17$ of age, before the division of the animals into the three experimental feeding groups, with that seen at 21 in MM-fed rat pups.

We also evaluated the effect of a reported inducer of precocious gut closure to $\mathrm{IgG}, \mathrm{HC}$, on macromolecular permeability in $\mathrm{PH}$ and S-fed rat pups $(1,15)$. At $14 \mathrm{~d}$ of age, pups were injected intraperitoneally with $5 \mathrm{mg}$ of $\mathrm{HC}$. At 17 d of age, the HCtreated pups were divided into two formula feeding groups ( $\mathrm{PH}$ $+\mathrm{HC}$ and $\mathrm{S}+\mathrm{HC}$ ) for $4 \mathrm{~d}$, and then evaluated at $21 \mathrm{~d}$ (Fig. 1).

Macromolecular Absorption, Studies. Loop preparation. After the experimental feeding period, rat pups were anesthetized with urethane $(1.3 \mathrm{~g} / \mathrm{kg}$, intraperitoneally) and the abdominal cavity opened by a mid-ventral incision. A loop of jejunum distal to the Ligament of Treitz was isolated in vivo, maintaining vascularization of the segment. The intestinal loops were approximately $15 \mathrm{~cm}$ in length (for $n=36$, mean \pm SEM was $15.4 \pm$ $0.5 \mathrm{~cm} / \mathrm{loop}$ ). The jejunal loops were cannulated at their proximal and distal ends, washed with warm $\left(37^{\circ} \mathrm{C}\right)$ isotonic saline, and kept intraabdominally moist; animal rectal temperature was maintained at 36.5 to $38^{\circ} \mathrm{C}$ with electric heating pads. The loops were equilibrated for two $30-\mathrm{min}$ periods with $1.5 \mathrm{~mL}$ aliquots of isotonic $(140 \mathrm{mM}) \mathrm{NaCl}$, buffered with $5 \mathrm{mM}$ Tris- $\mathrm{HCl}$ (pH 7.3). This fluid volume $(1.5 \mathrm{~mL})$ was chosen because it filled the $15 \mathrm{~cm}$ loops without causing distension. Fluid collection from the loops was by displacement of the contents with two gentle $10 \mathrm{~mL}$ volumes of air (18). After the equilibration period, a preweighed $1.5 \mathrm{~mL}$ aliquot of $0.5 \mathrm{~g} / \mathrm{dL}$ HRP (type II, Sigma Chemical Co., St. Louis, MO) in Tris-buffered (pH 7.3) isotonic saline was gently infused into the loop while the exit port was left open. The exit port was then closed and the HRP-containing solution kept in the loop for $30 \mathrm{~min}$ within the abdominal cavity and covered by a warm, soaked gauze pad. At the end of the experimental period, the lumenal fluid was collected by two air displacements into preweighed test tubes.

Blood collection and serum preparation. Blood was obtained from the dorsal aorta at the lumbar bifurcation, avoiding hemolysis, in a heparinized, plunger-free, syringe barrel fitted with a heparinized 22-gauge 1 -inch needle $(19,20)$. Blood $(0.25-0.50$ $\mathrm{mL}$ ) was allowed to flow into the barrel and was then gently decanted into small test tubes. The perfused intestinal loop was excised and its length measured under a 3-g tension. At the end of the experiment, the animals were killed by exsanguination. The blood was then transferred to Wintrobe tubes and centrifuged at $500 \times g$ on a clinical table top centrifuge (IEC, Needham Heights, MA) for $2 \mathrm{~h}$, yielding a light straw-colored serum.

Peroxidase activity assay. Assays were done immediately after centrifugation. A test run, however, did indicate that serum HRP activity is unaffected by overnight storage at $4^{\circ} \mathrm{C}$. Serum HRP was assayed as previously described $(19,20)$ by measuring the rate of change in absorbance produced by the oxidation of o-dianisidine at $460 \mathrm{~nm}$ over a $1-$ min period on a recording spectrophotometer (Shimatsu Electronics model UV-160, Tokyo, Japan). Fifty $\mu \mathrm{L}$ of serum were used for the assay. The units of activity were expressed as $\mu \mathrm{mol}$ hydrogen peroxide decomposed $/ \mathrm{mL}$ serum $\times \min \times \mathrm{cm}$ loop. We have previously demonstrated that enzyme activity in the range we detected is proportional to the actual concentration of HRP (19).

Because $\mathrm{Hb}$ is peroxidatic, we assessed possible hemolysis in our technique of serum preparation. S-fed pups were perfused in the absence of HRP and serum peroxidase activity determined. In these HRP-free pups, endogenous serum peroxidase activity was $38.2 \pm 29.3 \mathrm{IU} / \mathrm{mL} \times \mathrm{cm} \times$ min; less than MM controls exposed to the tracer, and similar to levels found in our earlier HRP-free control studies (19).

Net Water Transport. Net water absorption was evaluated gravimetrically by determining the difference between fluid recovered in tared test tubes and the amount infused into the loops and expressed as $\mu \mathrm{L}$ water absorbed $/ \mathrm{min} \times \mathrm{cm}$ loop (18).

Morphological Studies and Cylochemistry. To study the route of HRP penetration across the mucosa, samples were fixed in 